\title{
Channel electron bubbles in low-temperature helium gas
}

\author{
Yu.P. Monarkha \\ B. Verkin Institute for Low Temperature Physics and Engineering of the National Academy of Sciences of Ukraine \\ 47 Nauky Ave., Kharkiv 61103, Ukraine \\ E-mail: monarkha@ilt.kharkov.ua
}

Received March 23, 2018, published online August 28, 2018

\begin{abstract}
Self-localization of electrons caused by their interaction with helium vapor atoms in quasi-one-dimensional conducting channels over liquid helium is theoretically studied. We have shown that channel electron bubbles of a large size can exist at temperatures which are substantially lower than the critical temperature reported previously for electron bubbles of three-dimensional and two-dimensional electron systems. The transition from a large-size bubble to a small-size bubble is shown to be discontinuous, and a sort of bistability involving metastable states is described. The analysis given explains a sharp decrease of electron mobility in quasi-one-dimensional channels on liquid helium observed at $T \gtrsim 2,5 \mathrm{~K}$.
\end{abstract}

Keywords: electron bubble states, helium gas, self-localization, surface electrons, conducting channels.

\section{Introduction}

Interaction of a free electron with a collection of helium atoms leads to a remarkable self-localization effect: a localized electron and a strong reduction of the helium-gas density in the vicinity of the electron form a self-sustained complex called electron bubble [1]. The "bubble" concept explained a sharp decrease of the electron mobility in a dense helium gas with $n_{g} \geq n_{c}^{(3 \mathrm{D})} \sim 10^{21} \mathrm{~cm}^{-3}(T>3 \mathrm{~K})$, and helped with interpretation of electron and ion mobilities in liquid helium. In the presence of a strong magnetic field, the existence of large radius ions was shown [2] to be possible at $n_{g}<n_{c}^{(3 \mathrm{D})}$. The description of these ions is similar to the description of one-dimensional (1D) polarons: any interaction forming a potential well leads to a bound state. At $T \gtrsim 2.5 \mathrm{~K}$, the typical electron interaction energy with helium vapor $U_{0}$ is much larger than temperature, therefore, even a small reduction of the gas density can cause self-localization.

Ions of a large radius coupled to a helium gas density deformation can exist also in a two-dimensional (2D) electron system formed on the free surface of liquid helium (or other cryogenic substrates), if a strong magnetic field is applied perpendicularly to the interface [3]. In this case electron localization is induced by external fields, while electron interaction with the deformation of gas density just eliminates the degeneracy of the electron spectrum in the magnetic field. In the absence of the magnetic field, 2D electron bubbles in helium gas reportedly [4] can appear only if $n_{g}$ exceeds a critical value $n_{c}^{(2 D)}$ dependent on the strength of electron localization in the perpendicular direc- tion. The theory of these cavities was used to explain localization effects reported for 2D electrons on solid hydrogen [5] where $n_{c}^{(2 \mathrm{D})}$ is much less than $n_{c}^{(3 \mathrm{D})}$. Still, for electrons bound to the free surface of liquid helium, the critical temperature necessary for self-localization $T_{C}^{(2 D)}$ estimated [6] for the strong coupling regime appears to be close to that of the 3D system $\left(T_{c}^{(2 \mathrm{D})} \approx 3 \mathrm{~K}\right)$.

Recently, a strong mobility decrease of electrons trapped in quasi-one-dimensional channel states over liquid helium was observed [5] at comparatively low temperatures $T>2 \mathrm{~K}$. This interesting behavior was attributed to self-localization of channel electrons in dense helium vapor. A theoretical analysis and numerical calculations [6] performed for the strong coupling regime indicated that the critical temperature $T_{C}^{(1 D)}$ at which self-localization of channel electrons can appear slightly exceeds $3 \mathrm{~K}$, and it depends very weakly on the pressing electric field $E_{\perp}$. It should be noted that the relatively small difference in the critical temperatures found theoretically and in the experiment (about $0.5 \mathrm{~K}$ ) leads to a substantial difference in critical vapor densities (about two times) because the saturated gas density exponentially depends on $T$. The origin of this difference requires a theoretical explanation.

In strongly bound self-localized states [6], the electron localization length along the channel $\left(L_{x}\right)$ was shown to be surprisingly very close to the localization length in the transverse direction $\left(L_{y}\right)$. These remarkable results are in contrast with naive expectations triggered by the theory of large radius ions [2] which yields no density threshold and $L_{x} \gg L_{y}$. Therefore, an additional theoretical analysis of 
electron self-localization in quasi-one-dimensional conducting channels over liquid helium is necessary to explain the differences in theoretical results reported previously and describe the strong dependence of $T_{c}^{(1 \mathrm{D})}$ on the width of the conducting channel observed in the experiment [6].

In this work, following $[4,6]$, we consider a theoretical model which assumes strong electron localization in the direction perpendicular to the surface caused by the image potential and pressing electric field. Contrary to the previous treatments [2,4], the in-plane localization parameters $L_{x}$ and $L_{y}$ are not initially restricted by the conditions $L_{x}=L_{y}$ or $L_{y}=L_{0}$, where $L_{0}$ is the transverse localization length in the absence of the vapor density deformation. The electron localization length in the perpendicular direction $L_{z}$ generally is also affected by the gas density deformation. This model allows us to obtain analytical equations which, in the weak coupling regime, describe bubble states of electrons with $L_{x} \gg L_{y}$, similar to large radius ions [2] (here we still use the term "bubble state" even if the real gas density reduction is small). It is important that these states can exist at temperatures which are substantially lower than $3 \mathrm{~K}$ which explains experimental observations. At the same time, in the strong coupling regime, our equations yield $L_{x} \simeq L_{y}$ in accordance with numerical calculations of Ref. 6. Remarkably, the transition between solutions of the weak and strong coupling regimes is discontinuous, and a sort of bistability involving metastable states is predicted. Therefore, channel electron bubbles represent an interesting case for the theory of polaron systems [7-9].

\section{Free energy and basic equations}

In the experiment [6], conducting channels with electrons are formed above a liquid helium surface curved by capillary forces between glass light guides. The electron wave function in the channel will be considered as $\Psi(\mathbf{R})=f(z) \varphi(y) \psi(x)$, where $f(z)=2 \gamma^{3 / 2} z \exp (-\gamma z)$ describes electron localization in the direction perpendicular to the surface of liquid helium. In the limit of weak coupling with the vapor atom density, $\gamma \simeq \gamma_{1}$, where $\gamma_{1}$ is determined by the image potential $(-\Lambda / z)$ and the electric field $E_{\perp}$. The parameter $1 / \gamma_{1}$ represents the typical localization length in $z$-direction $L_{z} \approx 10^{-6} \mathrm{~cm}$. For the strong coupling regime, one have to consider an increase in $\gamma$ caused by the gas density deformation. The wave function $\varphi(y)$ is affected by the transverse confining potential $m \omega_{0}^{2} y^{2} / 2$ and by the redistribution of vapor atoms, while $\psi(x)$ describes an electron state along the channel.

In order to investigate self-localization of channel electrons, consider the free energy of an electron bubble $F$ which consists of the electron contribution $F_{\mathrm{el}}$, the free energy of helium gas $F_{\text {gas }}$, and the interaction term $F_{\text {int }}$. According to the conventional treatment $[1,2,4,6]$, the electron free energy

$$
F_{\mathrm{el}}=\int\left\{\frac{\hbar^{2}}{2 m}\left|\nabla_{\mathbf{r}} \psi \varphi\right|^{2}+\frac{m \omega_{0}^{2} y^{2}}{2}|\psi \varphi|^{2}\right\} d^{2} \mathbf{r}+F_{\perp}(\gamma),(1)
$$

where $\mathbf{r}=(x, y)$ is a $2 \mathrm{D}$ vector, $m$ is the electron mass, in the absence of the vapor density reduction inside the bubble, the frequency $\omega_{0}$ determines the transverse localization length $L_{0}=\sqrt{\hbar / m \omega_{0}}$, and

$$
F_{\perp}(\gamma)=\frac{\hbar^{2} \gamma^{2}}{2 m}-\Lambda \gamma+\frac{3 e E_{\perp}}{2 \gamma}
$$

represents the free energy of an electron due to its motion in the perpendicular direction. The function $f(z)$ does not enter the integrand of Eq. (1) because of the normalization condition $\int|f(z)|^{2} d z=1$, still it was used for obtaining $F_{\perp}(\gamma)$.

Considering the collection of vapor atoms as an ideal gas, we can define the free energy of vapor atoms as

$$
F_{\text {gas }}=\int n T \ln [g(T) n] d^{3} \mathbf{R},
$$

where $\mathbf{R}=(\mathbf{r}, z)$ is a 3D vector, $n(\mathbf{R})$ is the local gas density,

$$
g(T)=\frac{1}{\mathrm{e}}\left(\frac{2 \pi \hbar^{2}}{M T}\right)^{3 / 2},
$$

e is Euler's number, and $M$ is the mass of a helium atom. The interaction term depends on the electron wave function

$$
F_{\text {int }}=\frac{2 \pi \hbar^{2} a_{0}}{m} \int n(\mathbf{R})|\Psi(\mathbf{R})|^{2} d^{3} \mathbf{R},
$$

where $a_{0} \simeq 0.62 \cdot 10^{-8} \mathrm{~cm}$ is the scattering length.

The helium gas density deformation is determined by a sort of barometric formula [1]

$$
n=n_{g} \exp \left[-\frac{U_{0}}{n_{g} T}|\Psi(\mathbf{R})|^{2}\right]
$$

originating from variation of $F$ with respect to $n$. Here $n_{g}$ is the equilibrium density and

$$
U_{0}=\frac{2 \pi \hbar^{2} a_{0} n_{g}}{m}
$$

is the typical interaction energy. Using Eq. (4), $F_{\text {gas }}+F_{\text {int }}$ can be transformed into

$$
\begin{aligned}
& F_{\text {gas }}+F_{\text {int }}=n_{g} T \ln \left[g(T) n_{g}\right] \times \\
& \times \int \exp \left(-\frac{U_{0}}{n_{g} T}|\Psi(\mathbf{R})|^{2}\right) d^{3} \mathbf{R},
\end{aligned}
$$

where $\ln \left[g(T) n_{g}\right]<0$. For saturated pressure, $\ln \left[g(T) n_{g}\right]=-(1+Q / T)$, where $Q$ is the vaporization energy. 
Self-localization can appear when a change of the free energy caused by localization $\Delta F=F\left(L_{x}, L_{y}, \gamma\right)-$ $-F\left(\infty, L_{0}, \gamma_{1}\right)$ becomes negative (here $L_{x}$ and $L_{y}$ are localization lengths in the in-plane directions, and $\gamma \rightarrow \gamma_{1}$ if the gas density deformation is absent). Taking into account that the first two terms of the series expansion of Eq. (6) are independent of $L_{x}, L_{y}$, and $\gamma$, one can represent $\Delta F_{\text {gas }}+\Delta F_{\text {int }}$ as

$$
\begin{gathered}
\Delta F_{\text {gas }}+\Delta F_{\text {int }}=-n_{g} T\left|\ln \left[g(T) n_{g}\right]\right| \times \\
\times \int\left[\exp \left(-\frac{U_{0}}{n_{g} T}|\Psi|^{2}\right)-1+\frac{U_{0}}{n_{g} T}|\Psi|^{2}\right] d^{3} \mathbf{R} .
\end{gathered}
$$

This correction is responsible for the energy gain caused by localization.

In order to obtain $\Delta F_{\mathrm{el}}$ as a function of the localization parameters, we shall use the following trial wave functions

$$
\begin{aligned}
& \varphi(y)=\frac{1}{\pi^{1 / 4} L_{y}^{1 / 2}} \exp \left(-\frac{y^{2}}{2 L_{y}^{2}}\right), \\
& \psi(x)=\frac{1}{\pi^{1 / 4} L_{x}^{1 / 2}} \exp \left(-\frac{x^{2}}{2 L_{x}^{2}}\right),
\end{aligned}
$$

and assume that $f(z)$ has the same shape as in the absence of the gas density deformation (still, the actual value of $\gamma$ to be found by variation). In this treatment, $\Delta F_{\mathrm{el}}=F_{\mathrm{el}}\left(L_{x}, L_{y}, \gamma\right)-F_{\mathrm{el}}\left(\infty, L_{0}, \gamma_{1}\right)$ has a simple form

$$
\begin{gathered}
\Delta F_{\mathrm{el}}=\frac{\hbar^{2}}{4 m L_{x}^{2}}+\frac{\hbar^{2}}{4 m L_{y}^{2}}+\frac{m \omega_{0}^{2} L_{y}^{2}}{4}-\frac{\hbar^{2}}{2 m L_{0}^{2}}+ \\
+F_{\perp}(\gamma)-F_{\perp}\left(\gamma_{1}\right) .
\end{gathered}
$$

The fourth term in the right side of Eq. (10) represents the energy of zero-point vibrations $\hbar \omega_{0} / 2$ extracted. Obviously, the right side of Eq. (10) becomes zero when $L_{X} \rightarrow \infty$, $L_{y} \rightarrow L_{0}$ and $\gamma \rightarrow \gamma_{1}$. A finite $L_{x}$ together with $L_{y}<L_{0}$ and $\gamma>\gamma_{1}$ only increase the positive contribution $\Delta F_{\mathrm{el}}$.

For the trial wave functions of Eqs. (8) and (9), the contribution $\Delta F_{\text {gas }}+\Delta F_{\text {int }}$ given in Eq. (7) can be represented as

$$
\Delta F_{\text {gas }}+\Delta F_{\text {int }}=-\frac{3}{2^{5}} U_{0}\left|\ln \left[g(T) n_{g}\right]\right| G\left(\frac{R_{G}^{2} \tilde{\gamma}}{L_{x} L_{y}}\right)
$$

where $\tilde{\gamma}=\gamma / \gamma_{1}, R_{G}^{2}=2 \hbar^{2} a_{0} \gamma_{1} / m T$ and

$$
G(x)=\frac{2^{4}}{3} \sum_{n=1}^{\infty} \frac{(-1)^{n+1}(2 n+2) !}{(n+1) !(n+1)^{2 n+4}} x^{n} .
$$

The function $G(x)$ differs from the similar function $\Phi(x)$ defined previously [6] only by the normalization factor
$2^{5} / 3$ introduced for further convenience. The length parameter $R_{G}$ has a weak dependence on temperature and usually it is very small: $R_{G}(2.5 \mathrm{~K}) \simeq 2.5 \cdot 10^{-7} \mathrm{~cm}$ for $E_{\perp}=100 \mathrm{~V} / \mathrm{cm}$, and $R_{G}(2.5 \mathrm{~K}) \simeq 3.1 \cdot 10^{-7} \mathrm{~cm} \quad$ for $E_{\perp}=2000 \mathrm{~V} / \mathrm{cm}$.

Variation of $\Delta F$ with respect to $\tilde{\gamma}, L_{X}$, and $L_{y}$ yields three equations containing the derivative $G^{\prime}\left(R_{G}^{2} \tilde{\gamma} / L_{x} L_{y}\right)$. This derivative can be expressed from the equation $\partial \Delta F / \partial L_{X}=0$ and inserted into equations $\partial \Delta F / \partial \tilde{\gamma}=0$ and $\partial \Delta F / \partial L_{y}=0$. This procedure yields two simple relationships:

$$
\begin{gathered}
\tilde{\gamma}^{3}-\frac{\gamma_{0}}{\gamma_{1}} \tilde{\gamma}^{2}-\frac{1}{2 \gamma_{1}^{2} L_{0}^{2} l_{x}^{2}} \tilde{\gamma}-\frac{\gamma_{E}^{3}}{\gamma_{1}^{3}}=0, \\
l_{x}^{2}=\frac{l_{y}^{2}}{1-l_{y}^{4}},
\end{gathered}
$$

where we introduced two dimensionless localization lengths $l_{y}=L_{y} / L_{0}, l_{x}=L_{x} / L_{0}$, and two characteristic parameters $\gamma_{E}=\left(3 m e E_{\perp} / 2 \hbar^{2}\right)^{1 / 3}$ and $\gamma_{0}=m \Lambda / \hbar^{2}$. It should be noted that Eq. (14) was already given in Ref. 6. Equation (13) is a cubic equation whose solution can be found in an analytical form. Together with Eq. (14) it establishes the dependence $\tilde{\gamma}\left(l_{y}\right)$. It is worth noting also that the all parameters of these two equations do not depend on temperature, if we neglect the weak temperature dependence of the dielectric constant entering $\Lambda$.

Inserting the functions $l_{x}\left(l_{y}\right)$ and $\tilde{\gamma}\left(l_{y}\right)$ given above into the expression for $G^{\prime}\left(R_{G}^{2} \tilde{\gamma} / L_{x} L_{y}\right)$ we obtain the final equation for the dimensionless localization length $l_{y}$

$$
W_{\text {ch }}\left(l_{y}\right)=\kappa(T),
$$

where we introduced the following notations

$$
W_{\text {ch }}\left(l_{y}\right)=G^{\prime}\left(\frac{R_{G}^{2}}{L_{0}^{2}} \frac{\sqrt{1-l_{y}^{4}}}{l_{y}^{2}} \tilde{\gamma}\right) \frac{\tilde{\gamma}}{\sqrt{1-l_{y}^{4}}},
$$

and

$$
\kappa(T)=\frac{4 m T}{3 \pi n_{g}\left|\ln \left[n_{g} g(T)\right]\right| \hbar^{2} a_{0}^{2} \gamma_{1}} .
$$

The dimensionless parameter $\kappa$ depends strongly on temperature. It is large at low temperatures, and it becomes of the order of unity when $T \approx 2.6 \mathrm{~K}$. Obviously, it decreases with the pressing electric field because $\kappa \propto 1 / \gamma_{1}$.

The parameter $\kappa$ plays an important role in the description of self-localization of channel electrons and establishes its remarkable relationship with self-localization in the pure 2D electron system. For example, the characteristic temperature $T_{*}$ defined by the equation $\kappa(T)=1$ coincides with the critical temperature $T_{c}^{(2 D)}$ of self-localization in- 
duced by helium gas density in the pure 2D electron system $\left(\gamma\right.$ is fixed to $\left.\gamma_{1}\right)$ which is defined by the condition $n_{g}=n_{c}^{(2 \mathrm{D})}$, where [4]

$$
n_{c}^{(2 \mathrm{D})}=\frac{4 m T}{3 \pi\left|\ln \left[g(T) n_{g}\right]\right| \hbar^{2} a_{0}^{2} \gamma} .
$$

Therefore, formally we can write $\kappa=n_{c}^{(2 \mathrm{D})} / n_{g}$, though, as correctly stated in Ref. 6, for 2D electrons on liquid helium, it is impossible to fix $\gamma$ to $\gamma_{1}$, and the real critical parameters $T_{c}^{(2 \mathrm{D})}$ and $n_{c}^{(2 \mathrm{D})}$ are determined by different equations. In the pure 2D system, electron bubbles can appear only if $n_{g}>n_{c}^{(2 \mathrm{D})}$ when the parameter $\kappa$ is less than unity. There are no $2 \mathrm{D}$ electron bubbles at low temperatures where $\kappa(T)>1$. For channel electrons with the transverse confining potential, the possibility of existence of electron bubbles at $\kappa(T)>1$ requires a detailed investigation of Eq. (15).

The saturated vapor density is usually described by

$$
n_{g}=\left(M T / 2 \pi \hbar^{2}\right)^{3 / 2} \mathrm{e}^{-Q / T} .
$$

In calculations of the present work, the vaporization energy $Q$ is fixed to $7.36 \mathrm{~K}$ in order to fit the experimental data [10] on the saturated density of helium gas at $T \simeq 3 \mathrm{~K}$. An alternative formula [11] based on fitting experimental data [12] gives somewhat smaller values of $n_{g}$ if $T>2 \mathrm{~K}$.

\section{Channel ions of a large size}

First, it is reasonable to consider the limit of strong electron binding to the interface and to assume that at low enough $n_{g}$ the parameter $\gamma$ is not affected by the gas density deformation $(\tilde{\gamma}=1)$. This assumption is based on the fact that the third term of Eq. (13) is small if $\gamma_{1}^{2} L_{0}^{2} \gg 1$ and $l_{X}>1$. The 3D effects in electron self-localization will be described in the next Section. Under conditions of the experiment [6], $R_{G} \ll L_{0}$, and, therefore, the parameter $R_{G}^{2} / L_{0}^{2}$ entering the argument of $G^{\prime}$ in Eq. (16) is very small. Additionally, for large $\kappa(T)$ the left side of Eq. (15) can be compared with $\kappa$ only if $l_{y} \rightarrow 1$ when the argument of $G^{\prime}$ approaches zero $\left(G^{\prime} \rightarrow 1\right)$ while the factor $1 / \sqrt{1-l_{y}^{4}}$ entering $W_{\mathrm{ch}}\left(l_{y}\right)$ strongly increases. In this limiting case, Eq. (15) and Eq. (15) yield the simplest solution

$$
l_{y}=\left(1-\frac{1}{\kappa^{2}}\right)^{1 / 4}, l_{x}=\kappa l_{y} .
$$

If the system is cooling, the parameter $\kappa(T)$ increases and, therefore, $l_{y} \rightarrow 1\left(L_{y} \rightarrow L_{0}\right.$ ) while $l_{x}$ becomes much larger than unity $\left(L_{x} \gg L_{0}\right)$. In the opposite limit, when $\kappa(T) \rightarrow 1$, the both localization lengths $l_{y}$ and $l_{x}$ are rapidly shrinking.
The sharp decrease of $l_{y}$ at $T \rightarrow T_{*}$ means that the approximation $G^{\prime}(x) \simeq 1$ used for obtaining Eq. (19) fails in the vicinity of $T=T_{*}$. Since the next term in the sum of Eq. (12) has the opposite sign, it reduces the shrink effect. Thus, we have to consider a more accurate approximation: $G^{\prime}(x) \simeq 1-2^{8} 5 x / 3^{8}$. In this case, the omitted terms can only increase $G(x)$ and the effect of self-localization. Inserting this approximation of $G^{\prime}(x)$ into Eq. (16) we obtain a quartic equation for $l_{y}^{2}$ :

$$
l_{y}^{8}+2 \Omega l_{y}^{6}-\left(1-\frac{1}{\kappa^{2}}-\Omega^{2}\right) l_{y}^{4}-2 \Omega l_{y}^{2}-\Omega^{2}=0,
$$

where

$$
\Omega=\left(\frac{2}{3}\right)^{8} \frac{5 R_{G}^{2}}{\kappa L_{0}^{2}} .
$$

Using Eqs. (20) and (14) it is possible to find another useful relationship

$$
l_{x}=\left(1+\frac{\Omega}{l_{y}^{2}}\right) \kappa l_{y},
$$

valid only for the approximation $G^{\prime}(x) \simeq 1-2^{8} 5 x / 3^{8}$. This equation extends the asymptote of $l_{x}$ given in Eq. (19).

The temperature dependent parameter $\Omega$ is very small (usually $\Omega<0.02$ ). Still, it is necessary to keep it in Eq. (20) to extend the solution to the range $T \sim T_{*}$. The numerical evaluation of Eq. (20) is not difficult, still it is possible to find a simple analytical solution valid up to the point $T=T_{*}$. Neglecting the two terms proportional to $\Omega^{2}$ in Eq. (20) allows reducing this equation to a cubic equation whose solution can be represented as

$$
\begin{gathered}
l_{y}^{2}=\sqrt{1-\frac{1}{\kappa^{2}}} \frac{2 \cos (\beta)}{\sqrt{3}}-\frac{2 \Omega}{3}, \\
\beta=\frac{1}{3} \arccos \left(\frac{\sqrt{3}\left(2+1 / \kappa^{2}\right) \Omega}{\left(1-1 / \kappa^{2}\right)^{3 / 2}}\right) .
\end{gathered}
$$

Numerical calculations indicate that the analytical solution of Eq. (23) practically coincides with the exact solution of Eq. (20) even at $T \rightarrow T_{*}$. Similarly, it is easy to find also an analytical solution valid at $T>T_{*}$ but in this range soon one needs to consider the higher terms of the expansion series of Eq. (12).

The numerical solution of Eq. (20) is shown in Fig. 1 as a solid (blue) line. In this figure, the analytical solution given in Eq. (23) cannot be distinguished from the numerical solution up to $T=2.66 \mathrm{~K}$. As expected, the asymptote of Eq. (19) (dotted blue line) deviates from the solid line when the parameter $\kappa(T) \rightarrow 1$. It is noticeable also that at $T<T_{*}$ the localization length along the channel $l_{X}(T)$ (dashed red line) increases with lowering $T$ much faster 


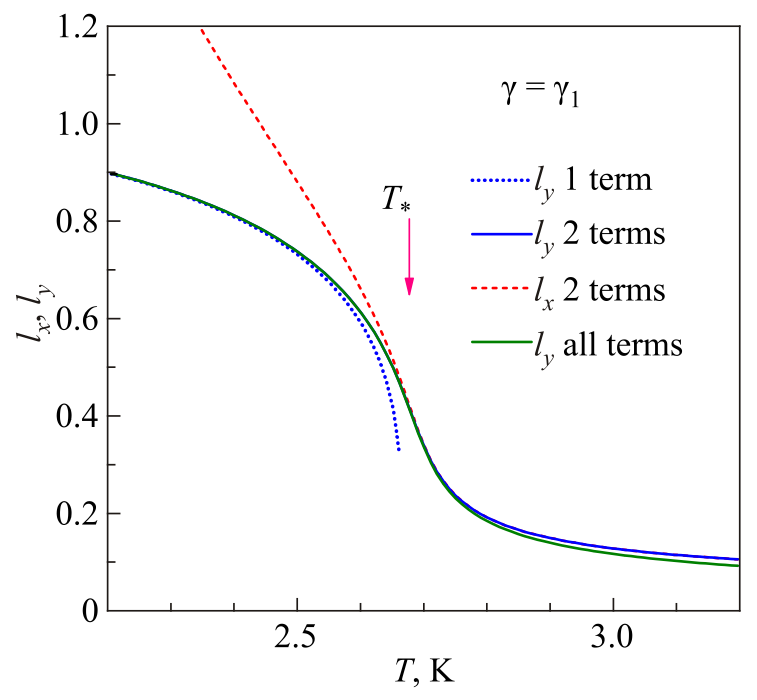

Fig. 1. (Color online) The dimensionless localization lengths $l_{x}$ and $l_{y}$ vs temperature calculated for $E_{\perp}=2000 \mathrm{~V} / \mathrm{cm}$, $L_{0}=0.2 \cdot 10^{-5} \mathrm{~cm}$ and different approximations: the asymptote of Eq. (19) based on the one-term approximation for $G(x)$ (dotted blue line), the solution of Eq. (20) based on the two-terms approximation for $G(x)$ [solid blue $\left(l_{y}\right)$ and dashed red $\left(l_{x}\right)$ ], the numerical solution of Eq. (15) using the exact form of $G(x)$ (solid olive line).

than $l_{y}(T)$ whose value is limited by 1 . The critical point $T=T_{*}$ is shown by the vertical pink arrow which separates the regimes of the weak and strong coupling.

The numerical solution of Eq. (15) including the all terms of the function $G(x)$ and using the assumption $\gamma=\gamma_{1}$ (electron localization in the vertical direction is not affected by the vapor density deformation) is shown in Fig. 1 as the solid (olive) curve. At low temperatures ( $T \leq T_{*}$ ) this solution coincides with the solid blue curve obtained employing only two first terms of the sum given in Eq. (12). In the opposite regime ( $T>T_{*}$ ), it is situated a bit lower than the numerical solution of Eq. (20). It is remarkable that in this regime the analytical solution of the reduced cubic equation (similar to that given in Eq. (23)) accidentally is close to the exact solution represented by the olive curve. Anyway, we can conclude that the first two terms of $G^{\prime}(x)$ give reasonable description of electron self-localization in a wide range of temperatures if $\gamma$ is fixed to $\gamma_{1}$.

With an increase of $L_{0}$ the transition range near $T=T_{*}$ becomes sharper, and in the limiting case $L_{0} \rightarrow \infty$, the solution of Eq. (20) transforms into the solution of the pure 2D problem [4]. Since the parameter $\kappa$ defined by Eqs. (17) and the critical density $n_{C}^{(2 D)}$ depend on the electron localization length in the perpendicular direction $L_{z} \approx \gamma_{1}^{-1}$, the critical temperature $T_{*}$ decreases with the pressing electric field $E_{\perp}$. Under typical experimental conditions, $T_{*}$ can be varied from about $3.2 \mathrm{~K}\left(E_{\perp}=100 \mathrm{~V} / \mathrm{cm}\right)$ to about $2.7 \mathrm{~K}\left(E_{\perp}=2000 \mathrm{~V} / \mathrm{cm}\right)$.
Inserting the solutions $l_{x}$ and $l_{y}$ obtained into $\Delta F=\Delta F_{\text {el }}+\Delta F_{\text {gas }}+\Delta F_{\text {int }}$ determined by Eqs. (10) and (11) we found that $\Delta F(T)<0$ for all temperatures. If $T=T_{*}$ and $L_{0}=0.2 \cdot 10^{-5} \mathrm{~cm}$, the absolute value $\left|\Delta F\left(T_{*}\right)\right| \approx 1 \mathrm{~K}$. The binding energy $|\Delta F(T)|$ decreases strongly with $L_{0}$ and for $L_{0}=10^{-4} \mathrm{~cm}$ it becomes very close to the binding energy of $2 \mathrm{D}$ electron bubbles obtained neglecting changes in $\gamma$ induced by localization:

$$
\Delta F^{(2 \mathrm{D})}=-\lambda \frac{\left|\ln \left[n_{g} g(T)\right]\right| n_{g} \hbar^{2} a_{0}}{4 m}\left(1-\frac{n_{c}^{(2 \mathrm{D})}}{n_{g}}\right)^{2},
$$

where $n_{g} \geq n_{c}^{(2 \mathrm{D})}$ and

$$
\lambda=\frac{\pi}{20}\left(\frac{3}{2}\right)^{9} \simeq 6 .
$$

Thus, the theory based on the assumption $\gamma=\gamma_{1}$ indicates that channel electron bubbles (their localization lengths and the free energy) continuously transform into pure 2D electron bubbles with a strong decrease in the transverse confining potential. On the other hand, Fig. 1 indicates that in the channel geometry, there is no a threshold for the electron bubble creation which differs from the result obtained for 2D bubbles [4,6]. Therefore, the electron bubbles of large size are possible in a channel geometry at low temperatures where the parameter $\kappa(T)$ is large.

\section{3D effects and self-localization bistability}

A very important result was obtained in Ref. 6: for electrons on liquid helium near $T_{C}^{(2 D)}$, the vapor density deformation affects crucially the electron localization length in the perpendicular direction $L_{z}$ and Eq. (24) becomes inapplicable to the electron system. This means that describing channel electron bubbles at $T \approx T_{*}$ we have to consider 3D effects and take into account changes of $\gamma$ induced by the gas density deformation.

In the general case, we need to solve Eq. (15) taking into account the increase of the localization parameter $\gamma$ induced by self-localization. Already the structure of Eq. (13) indicates that a substantial increase in $\tilde{\gamma}$ above the unity should lead to a strong decrease of $l_{x}$ and $l_{y}$. The cubic equation for $\tilde{\gamma}$ can be solved analytically

$$
\tilde{\gamma}=(q+\sqrt{P})^{1 / 3}+(q-\sqrt{P})^{1 / 3}+\frac{\gamma_{0}}{3 \gamma_{1}},
$$

where

$$
\begin{gathered}
q\left(l_{y}\right)=\frac{\gamma_{0}^{3}}{27 \gamma_{1}^{3}}+\frac{\gamma_{0}\left(1-l_{y}^{4}\right)}{12 \gamma_{1}^{3} L_{0}^{2} l_{y}^{2}}+\frac{\gamma_{E}^{3}}{2 \gamma_{1}^{3}}, \\
P\left(l_{y}\right)=q^{2}\left(l_{y}\right)-\frac{1}{27^{2}}\left[\frac{\gamma_{0}^{2}}{\gamma_{1}^{2}}+\frac{3\left(1-l_{y}^{4}\right)}{2 \gamma_{1}^{2} L_{0}^{2} l_{y}^{2}}\right]^{3} .
\end{gathered}
$$


The function $P\left(l_{y}\right)$ changes its sign at $l_{y} \equiv l_{c} \simeq 0.142$ (for $L_{0}=0.2 \cdot 10^{-5} \mathrm{~cm}$ ) becoming negative if $l_{y}<l_{c}$. For $P<0$, the solution has a trigonometric form similar to that found in the preceding Section for $l_{y}$ [Eq. (23)].

The dependence $\tilde{\gamma}\left(l_{y}\right)$ found above should be inserted into Eqs. (16). Thus, the final equation for $l_{y}$ has the form $W_{\mathrm{ch}}\left(l_{y}\right)=\kappa$, where $W_{\mathrm{ch}}\left(l_{y}\right)$ includes the all terms of $G^{\prime}(x)$. The solutions of this equation for three typical temperatures are illustrated in Fig. 2. As expected, the function $W_{\mathrm{ch}}\left(l_{y}, T\right)$ depends rather weakly on temperature (here we fixed dielectric constants of liquid helium and vapor to their values at $T=2.5 \mathrm{~K})$. Changing $T$ affects noticeably $W_{\mathrm{ch}}\left(l_{y}\right)$ only in the range of small $l_{y}\left(l_{y} \lesssim 0.2\right)$. At the same time, the parameter $\kappa(T)$ shown in Fig. 2 by three horizontal lines has a very strong dependence on $T$. Therefore, at a low temperature (for example, $T=2.25 \mathrm{~K}$ ) there is only one solution of the equation $W_{\mathrm{ch}}\left(l_{y}\right)=\kappa$ illustrated in the figure by the olive circle. At the chosen temperature $(T=2.25 \mathrm{~K})$ the intersection of the horizontal line $\kappa(2.25 \mathrm{~K})$ with $W_{\mathrm{ch}}\left(l_{y}\right)$ approximated by the assumption $\tilde{\gamma}=1$ (black dotted line) yields practically the same result for $l_{y}$.

Contrary to the curve calculated for $\tilde{\gamma}=1$, the exact function $W_{\mathrm{ch}}\left(l_{y}\right)$ is not monotonous, and in a certain temperature range there are three solutions of the equation $W_{\text {ch }}\left(l_{y}\right)=\kappa$, as shown in Fig. 2 for $T=2.56 \mathrm{~K}$. Only two of these solutions indicated by filled red circles represent minima of $\Delta F$. Therefore, in this temperature range, there is self-localization bistability, and the transition to the strong coupling regime is not continuous. At substantially higher temperatures, there is only one solution with a small

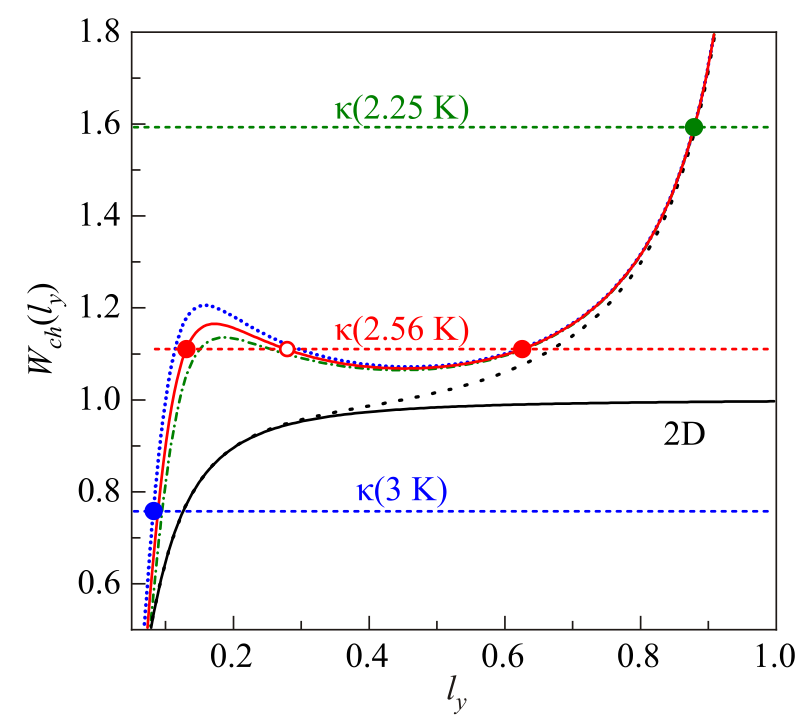

Fig. 2. (Color online) Graphical solution of the equation $W_{\mathrm{ch}}\left(l_{y}\right)=\kappa$ [Eq. (15)] for $L_{0}=0.2 \cdot 10^{-5} \mathrm{~cm}$ and three typical temperatures. The dashed horizontal lines represent $\kappa$ at different $T$. Their cross-points with curves representing $W_{\mathrm{ch}}\left(l_{y}\right)$ yield the solutions for $l_{y}$ shown by circles. The black dotted curve represents $W_{\mathrm{ch}}\left(l_{y}\right)$ for the approximation $\gamma=\gamma_{1}$. The black solid curve is calculated for the pure 2D system with $\gamma=\gamma_{1}$. $l_{y}$ indicated in the figure by the blue circle calculated for $T=3 \mathrm{~K}$. In this range, the approximate solution given by the intersection with the dotted curve deviates substantially from the exact solution.

The numerical solutions of the equation $W_{\mathrm{ch}}\left(l_{y}\right)=\kappa$ are shown in Fig. 3 as functions of temperature. In the limit of low temperatures, the high branch shown by the solid blue curve approaches the approximate solution obtained for the assumption $\tilde{\gamma}=1$ (dotted curve). The low branch shown by the solid red curve is quite distant from the dotted curve. The transition from the high branch to the low branch obviously cannot be continuous. Figure 3 indicates that there is a bistability range restricted by two vertical arrow-lines. Here we still use the term "bistability" in spite of the fact that at certain temperatures the branches can become metastable.

The free energy gain $\Delta F$ induced by self-localization is shown in Fig. 4 for different values of the transverse confining potential indicated by different $L_{0}$. The two branches are shown by separate lines of the same color. For $L_{0}=0.2 \cdot 10^{-5} \mathrm{~cm}$, the high and low branches (black solid lines) have a cross-point at about $2.54 \mathrm{~K}$. At higher temperatures, the high-branch line becomes metastable, and eventually it reaches the end-point. On the left side from the cross-point, the low-branch line is metastable and, moreover, soon $\Delta F$ becomes positive. With an increase of $L_{0}$ the cross-point shifts up and right as shown by dashed (blue, $L_{0}=0.3 \cdot 10^{-5} \mathrm{~cm}$ ) and dash-dotted (olive, $L_{0}=10^{-5} \mathrm{~cm}$ ) lines. The high-branch line eventually $\left(L_{0} \rightarrow \infty\right)$ approaches the zero line, while the low-branch line approaches the solid red line calculated for the 2D electron system. In should be noted that in Fig. 4, the low-branch line calculated for $L_{0}=10^{-5} \mathrm{~cm}$ cannot be distinguished from the red line calculated for $L_{0}=\infty$.

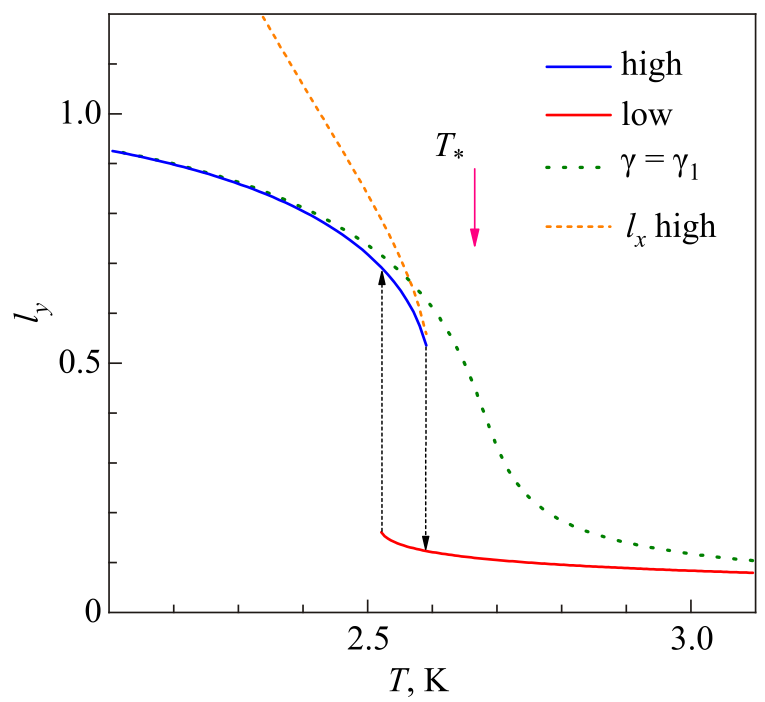

Fig. 3. (Color online) The numerical solutions of Eq. (15) $l_{y}(T)$ calculated for $L_{0}=0.2 \cdot 10^{-5} \mathrm{~cm}$. The dotted line represents the result of the approximation $\gamma=\gamma_{1}$. 


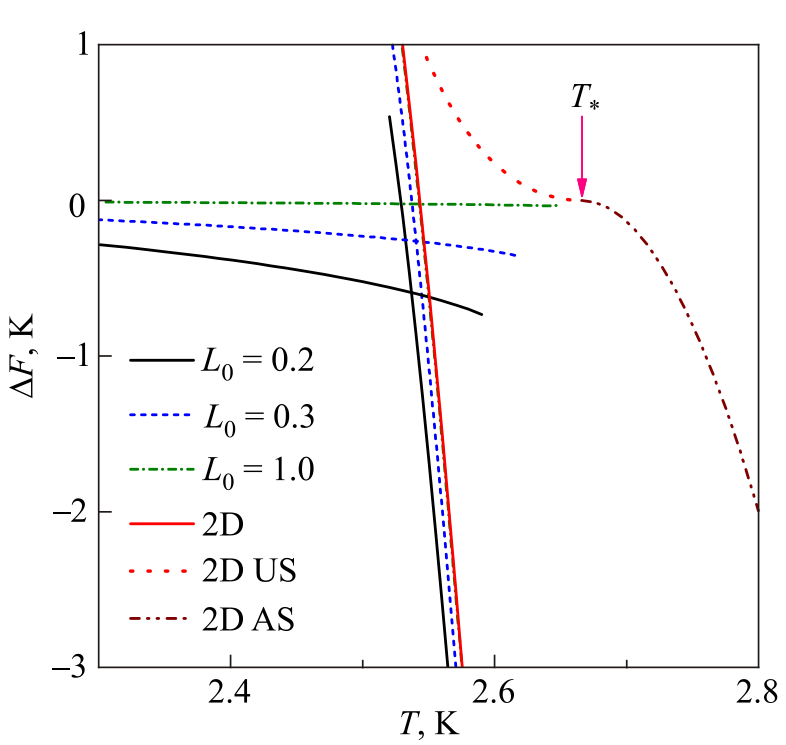

Fig. 4. (Color online) The free energy gain $\Delta F$ caused by selflocalization vs temperature calculated for different transverse confining potentials indicated in the legend by $L_{0}$ in units $10^{-5} \mathrm{~cm}$. The dotted red line represents the unstable (US) solution of the 2D electron system. The dash-dot-dot line represents the asymptotic solution [4] (AS) based on the assumption $\gamma=\gamma_{1}$ and given in Eq. (24).

The solid red line $\left(L_{0}=\infty\right)$ in Fig. 4 obtained using the all terms of the function $G(x)$ remarkably has nothing in common with the result of calculations based on the assumption $\gamma=\gamma_{1}$ and restricted by the first two terms of the sum defining $G(x)$ in Eq. (12) (wine dash-dot-dot line). This seems strange and requires additional explanations because, for a large in-plane localization radius $L \propto 1 / \sqrt{n_{g}-n_{c}}$ (this can also be seen from the solid black line of Fig. 2), the argument of the function $G(x)$ is small. This means that the usual self-verification procedure fails in this particular case.

The free energy $\Delta F$ of the $2 \mathrm{D}$ electron system with $\gamma$ affected by self-localization is described by two parameters: $\tilde{\gamma}$ and the in-plane localization radius $L$. These parameters satisfy two equations which are found similar to Eqs. (13) and (15):

$$
\begin{gathered}
\frac{1}{L^{2}}=\frac{2 \gamma_{1}^{2}}{\tilde{\gamma}}\left(\tilde{\gamma}^{3}-\frac{\gamma_{0}}{\gamma_{1}} \tilde{\gamma}^{2}-\frac{\gamma_{E}^{3}}{\gamma_{1}^{3}}\right), \\
W_{2 \mathrm{D}}(\tilde{\gamma}, L)=\kappa(T),
\end{gathered}
$$

where

$$
W_{2 \mathrm{D}}(\tilde{\gamma}, L)=\tilde{\gamma} G^{\prime}\left(\frac{R_{G}^{2} \tilde{\gamma}}{L^{2}}\right)
$$

and the parameters $\kappa(T)$ and $R_{G}$ are defined above. Remarkably, the expression for $W_{2 \mathrm{D}}$ can be also obtained directly from Eq. (16) for $W_{\mathrm{ch}}$ considering the limiting case
$L_{0} \rightarrow \infty$ which allows us to neglect $l_{y}^{4}$ in the square root expressions. Here, it is instructive to solve Eq. (29) with regard to the parameter $\tilde{\gamma}$ by inserting Eq. (28) into Eq. (30). The resulting equation $W_{2 \mathrm{D}}(\tilde{\gamma})=\kappa$ is solved numerically, and its solutions are shown in Fig. 4 by the solid and dotted red lines. The dotted line represents an unstable solution given here by way of illustration. The solid red line represents a stable solution obtained using the all terms of $G(x)$.

It is interesting that the solid red line of Fig. 4 is shifted substantially to the left from the result based on the asymptotic form of $G(x)$ and it does not approach the critical point $T_{*}$ defined above by the condition $n_{g}=n_{c}^{(2 \mathrm{D})}$. This strange situation is explained by Fig. 5 where the resulting function $W_{2 \mathrm{D}}(\tilde{\gamma})$ is shown for three different temperatures. It is very important that for electrons on liquid helium the function $W_{2 \mathrm{D}}(\tilde{\gamma})$ has a maximum in the range $\gamma / \gamma_{1}>1$. Therefore, when $\kappa(T)$ meets this maximum, 2D electron bubbles appear already with the parameter $\gamma$ which is substantially larger than $\gamma_{1}$ and, therefore, with a finite (!) radius $L$ determined by Eq. (28). In a certain temperature range, there are two solutions of the equation $W_{2 \mathrm{D}}(\tilde{\gamma})=\kappa$ shown in Fig. 5 by red circles. The left circle represents an unstable solution (here $L$ increases with $T$ ) which can reach the point $T_{*}$, as illustrated in Fig. 4 by the red dotted curve. The stable solution (the red filled circle) appears at $T<T_{*}$ and it eventually leads to $\Delta F<0$ (see the red solid line in Fig. 4).

It should be emphasized that for 2D electrons on liquid helium, the self-verification procedure of finding a solution of the equation $W_{2 \mathrm{D}}(L)=\kappa$ is not sufficient and it can bring to a wrong result. For example, assuming that at a critical point 2D electron bubbles appear with a large radi-

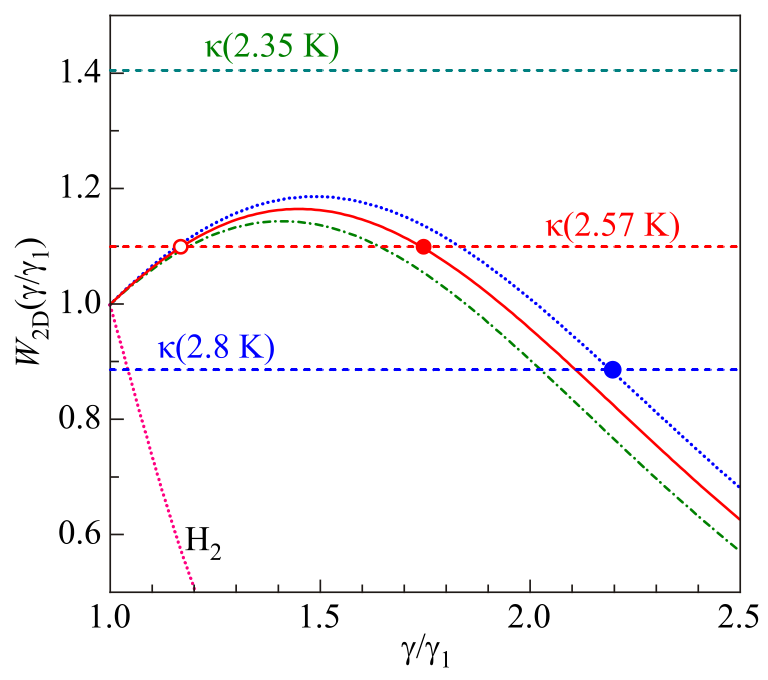

Fig. 5. (Color online) Graphical solution of the equation $W_{2 \mathrm{D}}(\tilde{\gamma})=\kappa$ describing $2 \mathrm{D}$ electron bubbles for $E_{\perp}=2000 \mathrm{~V} / \mathrm{cm}$. The dashed horizontal lines represent $\kappa$ at different $T$. Their cross-points with curves representing $W_{2 \mathrm{D}}(\tilde{\gamma})$ at three temperatures yield the solutions for $\tilde{\gamma}$ shown by circles. The dotted pink curve represents $W_{2 \mathrm{D}}(\tilde{\gamma})$ for electrons on solid hydrogen. 
us $L$, we seemingly can neglect $1 / L^{2}$ in Eq. (28), obtaining $\gamma=\gamma_{1}$, and disregard higher terms in the expansion series for $G^{\prime}(x)$. As a result, one obtains $1 / L^{2} \rightarrow 0$ when $n_{g} \rightarrow n_{c}^{(2 \mathrm{D})}$ which justifies the initial assumption. The later is valid also for the exact form of $G^{\prime}(x)$, as can be seen from the solid black curve (marked with 2D) in Fig. 2. Nevertheless, the accurate solution, taking into account 3D effects, at the critical point leads to a finite and rather small $L \simeq 3.4 \cdot 10^{-7} \mathrm{~cm}$, as follows from Fig. 5. This means that a sort of polaronic phase transition can exist in the 2D electron system on liquid helium.

We compared $W_{2 \mathrm{D}}$ as the function of $L$ (properly normalized) with the respective curve $W_{\text {ch }}\left(l_{y}\right)$ given in Fig. 2 and found that $W_{2 \mathrm{D}}\left(L / L_{0}\right)$ practically coincides with the solid red curve in the range $l_{y}<0.3$ which includes the maximum. At larger $L / L_{0}$, it decreases approaching unity from the upper side, in contrast with the solid black curve (2D) calculated for $\gamma=\gamma_{1}$.

Another important point is that the position of the maximum of $W_{2 \mathrm{D}}(\tilde{\gamma})$ strongly depends on $R_{G}$ and $\gamma_{1}$ shifting left when $\gamma_{1}$ increases. For the 2D electron system on liquid helium, $\gamma_{1}$ is relatively small, and, therefore, the maximum appears at $\gamma / \gamma_{1}>1$. The situation changes drastically for electrons on the surface of solid hydrogen. In this case, as shown by the pink dotted line of Fig. 5, the maximum is shifted into the unphysical range $\gamma<\gamma_{1}$, and a stable solution with a large radius $L$ appears at $\kappa(T)<1\left(T>T_{C}^{(2 D)}\right)$ in agreement with the approximation $\gamma=\gamma_{1}$ and the results of Ref. 4 .

It is instructive to compare $W_{2 \mathrm{D}}(\tilde{\gamma})$ with the similar function for channel electrons $W_{\mathrm{ch}}(\tilde{\gamma})$ which can be obtained from Eq. (16) expressing $l_{x}$ and $l_{y}$ as functions of $\tilde{\gamma}$.

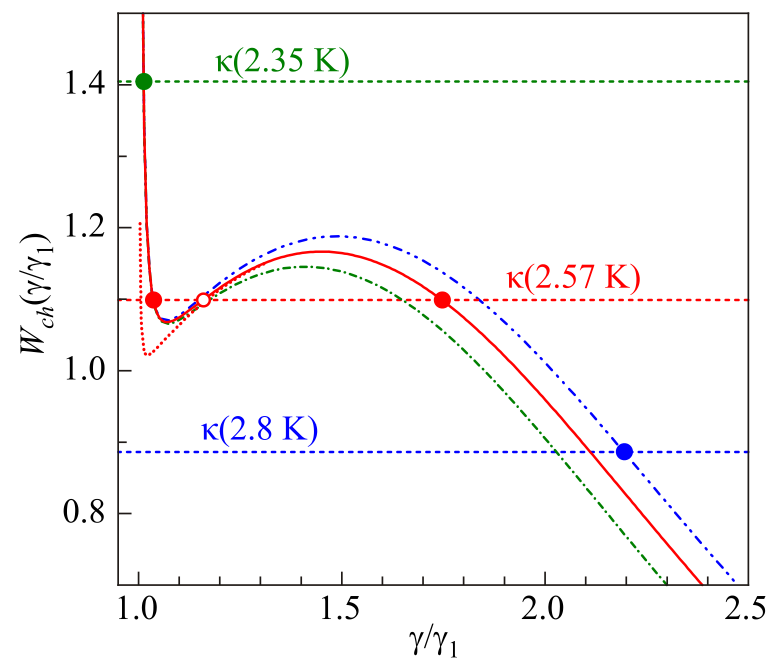

Fig. 6. (Color online) Graphical solution of the equation $W_{\mathrm{ch}}(\tilde{\gamma})=\kappa$ describing channel electron bubbles for $E_{\perp}=2000 \mathrm{~V} / \mathrm{cm}$ and $L_{0}=0.2 \cdot 10^{-5} \mathrm{~cm}$. The dashed horizontal lines represent $\kappa$ at different $T$. Their cross-points with curves representing $W_{\mathrm{ch}}(\tilde{\gamma})$ for three different temperatures yield the solutions for $\tilde{\gamma}$ shown by circles. The dotted red line is calculated for $L_{0}=0.5 \cdot 10^{-5} \mathrm{~cm}$.
Remarkably, $W_{\mathrm{ch}}(\tilde{\gamma})$ shown in Fig. 6 also has a maximum positioned approximately at the same value of $\tilde{\gamma}$. This means that self-localization bistability of channel electrons and and the appearance of $2 \mathrm{D}$ bubbles at a critical point with a finite radius have the same origin. The important difference is that $W_{\text {ch }}(\tilde{\gamma})$ does not approach unity when $\tilde{\gamma} \rightarrow 1$, but strongly increases which leads to a solution with $l_{y} \approx 1$. The preliminary analysis indicates that selflocalization bistability exists also for conducting channels with a symmetrical confining potential $U(r) \propto r^{2}$, and, therefore, it can be relevant also to large radius ions induced by a strong magnetic field [2].

The theory developed here, as well as the theories reported previously [4,6], disregards the correction to the free energy of helium gas proportional to $|\nabla n(\mathbf{r}, z)|^{2}$ which qualitatively can be considered as a sort of surface tension contribution. This term is expected to be small for bubbles of a large size, but in the opposite limit it can be substantial. This can affect the critical temperature for 2D electron bubbles. Anyway, the 2D model obviously cannot explain the strong dependence of the critical temperature on the strength of the confining potential observed in the experiment conducted using quasi-one-dimensional channels on liquid helium [6]. The strong decrease of $|\Delta F|$ with $L_{0}$ obtained here for the high-branch solution and shown in Fig. 5 correlates with these experimental results. Still, there is an interesting question whether self-localized states with $|\Delta F|$ substantially smaller than $T$ can affect the mobility of channel electrons. The experimental data [6] unambiguously prove that a sharp reduction of electron mobility exist also at $T \simeq 2.5 \mathrm{~K}$ for sufficiently large $L_{0} \gtrsim 0.5 \cdot 10^{-5} \mathrm{~cm}$. Under these conditions, $|\Delta F|$ is rather small as compared to $T$, and it seems that one can not expect a predominant population of bubble states at such low temperatures.

The electron-bubble states discussed here remind fluctuon states of electrons in disordered systems $[13,14]$ whose internal parameters can be easily changed. In these states, electrons are bound to a fluctuation of density or an internal parameter. If fluctuons can be considered as 3D quasiparticles, the ratio of equilibrium numbers of selflocalized electrons (fluctuons) and free electrons is proportional (besides the well-known exponential factor) to the mass factor $\left(M_{f} / m\right)^{3 / 2}$, where $M_{f}$ is the effective mass of a fluctuon [13]. Therefore, for channel electron bubbles with the effective mass $M_{b}$, one can expect that the ratio

$$
\frac{N_{b}}{N_{e}}=\sqrt{\frac{M_{b}}{m}} \exp \left(-\frac{\Delta F}{T}\right),
$$

where $N_{b}$ and $N_{e}$ are the number of electron bubbles and free electrons, respectively. Assuming that the bubble effective mass $M_{b}$ is much larger than the free electron mass $m$, we conclude that the ratio $N_{b} / N_{e}$ can be large even for a small binding energy $|\Delta F|$. In this case, the transition temperature observed in the experiment can be associated 
with a sharp increase of the bubble effective mass. Additionally, it should be noted that channel electrons on liquid helium is a highly correlated system, therefore, even a small fraction of $N_{b}$ can substantially reduce conductivity of the entire system.

\section{Conclusions}

In this work, we presented the theoretical analysis of bubble electron states caused by a dense helium gas in quasi-one-dimensional conducting channels over liquid helium. The theory reported unambiguously proves that in addition to channel ions of a small size found previously [6] there is a possibility of existence of channel electron bubbles of a large size under usual experimental conditions. These two solutions are shown to be separate, and in the transition regime there is a sort of self-localization bistability involving metastable states. We established a remarkable relationship between the bistability of channel electron bubbles and the unusual onset of the 2D bubble creation with a finite radius on the free surface of liquid helium. These theoretical results explain experimental observations of self-localized electron states in conducting channels over liquid helium at relatively low temperatures ( $T \simeq 2.5 \mathrm{~K})$ [6]. The free energy of electron bubbles of a large size is shown to depend strongly on the strength of the transverse confining potential which qualitatively agrees with the experimental dependence of the critical temperature on the radius of curvature of liquid helium in the conducting channels [6].

The author acknowledges valuable discussions with S.S. Sokolov.

1. J. Levin and T. Sanders, Phys. Rev. 154, 138 (1967).

2. L.S. Kukushkin and V.B. Shikin, Zh. Eksp. Teor. Fiz. 63, 1830 (1972) [Sov. Physics JETP 36, 969 (1973)].

3. Yu.P. Monarkha, Fiz. Nizk. Temp. 1, 1322 (1975) [Sov. J. Low Temp. Phys. 1, 634 (1975)].

4. V. Shikin, J. Low Temp. Phys. 139, 503 (2005).

5. P. Adams, Phys. Rev. Lett. 65, 3333 (1990).

6. V.A. Nikolaenko, A.V. Smorodin, and S.S. Sokolov, Fiz. Nizk. Temp. 37, 119 (2011) [Low Temp. Phys. 37, 95 (2011)].

7. B. Gerlach and H. Löwen, Rev. Mod. Phys. 63, 63 (1991).

8. Yu.P. Monarkha and K. Kono, Phys. Rev. B 65, 212507 (2002).

9. Yu.P. Monarkha and K. Kono, Two-Dimensional Coulomb Liquids and Solids, Springer-Verlag, Berlin (2004).

10. Brookhaven National Laboratory Selected Cryogenic Data Notebook, V. I, Section II (Properties of helium), compiled and edited by J.E. Jensen, W.A. Tuttle, R.B. Stewart, H. Brechna, and A.G. Prodell, United States of America, (1980).

11. S.S. Sokolov, G.-Q. Hai, and N. Studart, Phys. Rev. B 51, 5977 (1995).
12. B.N. Esel'son, V.N. Grigor'ev, V.G. Ivantsov, and E.Ya. Rudavskii, Properties of Liquid and Solid Helium Izdatel'stvo Standartov, Moscow (1978).

13. M.A. Krivoglaz, Fiz. Tverd. Tela 11, 2230 (1969) [Sov. Physics Solid State 11, 1802 (1970)].

14. M.A. Krivoglaz, Uspekhi Fiz. Nauk 111, 617 (1973) [Sov. Phys. Uspekhi 16, 856 (1974)].

\section{Пузиркові стани електронів в провідних каналах за наявності геліевого газу}

\section{Ю.П. Монарха}

Теоретично досліджено автолокалізацію електронів в квазіодновимірних провідних каналах над рідких геліем, викликана іх взаемодією з атомами геліевого газу. Показано, що в таких каналах пузиркові стани електронів великого розміру можуть існувати при температурах, істотно менших, ніж критичні температури, знайдені раніше для електронних пузирків в тривимірних і двовимірних системах. Показано також, що перехід від пузиркових станів великого розміру до пузиркових станів малого розміру не $\epsilon$ безперервним, що означае наявність метастабільних станів і свого роду бістабільності системи. Приведений теоретичний аналіз пояснює різке падіння рухливості електронів в квазіодновимірних каналах над рідким геліем, виявлене при $T \gtrsim 2,5 \mathrm{~K}$.

Ключові слова: пузиркові стани електронів, геліевий газ, автолокалізація, поверхневі електрони, провідні канали.

\section{Пузырьковые состояния электронов в проводящих каналах при наличии гелиевого газа}

\section{Ю.П. Монарха}

Теоретически исследована автолокализация электронов в квазиодномерных проводящих каналах над жидких гелием, вызванная их взаимодействием с атомами гелиевого газа. Показано, что в таких каналах пузырьковые состояния электронов большого размера могут существовать при температурах, существенно меньших, чем критические температуры, найденные ранее для электронных пузырьков в трехмерных и двумерных системах. Показано также, что переход от пузырьковых состояний большого размера к пузырьковым состояниям малого размера не является непрерывным, что подразумевает наличие метастабильных состояний и своего рода бистабильности системы. Приведенный теоретический анализ объясняет резкое падение подвижности электронов в квазиодномерных каналах над жидким гелием, обнаруженное при $T \gtrsim 2,5 \mathrm{~K}$.

Ключевые слова: пузырьковые состояния электронов, гелиевый газ, автолокализация, поверхностные электроны, проводящие каналы. 